\title{
PUBERDADE PRECOCE: CONDIÇÕES ASSOCIADAS
}

Precocious puberty: associated conditions

Pubertad precoz: condiciones asociadas

Artigo Original

\section{RESUMO}

Objetivo: Analisar as características das crianças com puberdade precoce central (PPC), identificando-se tanto suas características clínico-epidemiológicas como os fatores determinantes para o recebimento de uma atenção à saúde oportuna. Métodos: Estudo retrospectivo e analítico de 342 prontuários, entre 1994 e 2010, de crianças de 1 a 11 anos com PPC, provenientes do ambulatório de endocrinologia de um hospital de referência. Analisaram-se as variáveis biológicas, econômicas, familiares e nutricionais de forma uni e bivariada, utilizando-se o teste Qui-Quadrado na avaliação estatística das associações. Resultados: Identificaram-se como principais características: 25 (7,3\%) eram adotadas, 92 $(27,1 \%)$ estavam com sobrepeso, $85(25,1 \%)$ estavam com obesidade, e $205(60,3 \%)$ tiveram início da puberdade antes dos 6 anos. Crianças com PPC atendidas tardiamente foram as que apresentaram sinais da puberdade mais cedo $(\mathrm{p}<0,001)$ e pertenciam a famílias de menor renda $(p<0,002)$. Conclusão: Elevadas proporções de crianças com PPC eram adotadas e portadoras de sobrepeso/obesidade. Crianças com aparecimento excessivamente precoce dos sinais de puberdade e de baixa renda familiar sofreram significativo retardo na obtenção da atenção especializada.

Descritores: Puberdade Precoce; Obesidade Pediátrica; Saúde Pública.

\section{ABSTRACT}

Objective: To analyze the characteristics of children with central precocious puberty (CPP), identifying both their clinical and epidemiological characteristics, as well as the determinants for receiving timely specialized health care. Methods: Cross-sectional retrospective study with analytical approach of 342 medical records of children aged 1 to 11 years with CPP, from the Endocrinology Outpatient Clinic of a reference hospital. Biological, socio-economic, family and nutritional data of the 1994 to 2010 period was submitted to univariate and bivariate analysis, using the chi-square test for the statistical evaluation of the associations. Results: The main features of children with CPP were: being adopted, 25 (7.3\%); being overweight, 92 (27.1\%); being obese, 85 (25.1\%); initiating puberty before six years of age, 205 (60.3\%). Children with CPP who received late specialized care were the ones who most precociously presented signs of puberty $(p<0.001)$, and belonged to families with lower income $(p<0.002)$. Conclusion: High proportions of children with CPP were adopted and presented overweight/obesity. Children with excessively early onset of puberty signs, and belonging to low income families, suffered significant delay in obtaining specialized care.

Descriptors: Puberty, Precocious; Pediatric Obesity; Public Health.

\section{RESUMEN}

Objetivo: Analizar las características de los niños con pubertad precoz central (PPC) identificando sus características clínico-epidemiológicas como factores determinantes para el recibimiento de una atención a la salud oportuna. Métodos: Estudio transversal, retrospectivo y analítico de 342 historiales clínicos de niños entre 1 y 11 años con PPC del ambulatorio de endocrinología de un hospital de referencia entre 1994 y 2010. Las variables biológicas, económicas, familiares y nutricionales fueron analizadas de forma uni y bivariada utilizando la prueba del Chi-cuadrado en la evaluación estadística de las asociaciones. Resultados: Las principales características identificadas fueron: 25 (7,3\%) eran adoptados, 92 (27,1\%) tenían sobrepeso, 85 (25,1\%) eran obesos y 205 (60,3\%)

\section{Celso Junior Vanderley Cavalcante $^{(1)}$ \\ Luciano Lima Correia ${ }^{(1)}$ Durval Damiani( ${ }^{(2)}$}

\author{
1) Universidade Federal do Ceará - UFC - \\ Fortaleza (CE) - Brasil \\ 2) Universidade de São Paulo - USP - São \\ Paulo (SP) - Brasil
}

Recebido em: 30/01/2013 Revisado em: 11/07/2013

Aceito em: 26/02/2014 
iniciaron la pubertad antes de los 6 años. Niños con PPC con retraso en la asistencia presentaron señales de pubertad más precoz $(p<0,001) y$ pertenecian a familias con menor renta $(p<0,002)$. Conclusión: Elevadas proporciones de niños con PPC eran adoptados y portadores de sobrepeso/obesidad. Niños con el surgimiento excesivamente precoz de señales de pubertad y baja renta familiar sufrieron retraso significativo en la obtención de la asistencia especializada.

Descriptores: Pubertad Precoz; Obesidad Pediátrica; Salud Pública.

\section{INTRODUÇÃO}

Puberdade precoce é definida como o aparecimento dos sinais físicos de desenvolvimento puberal em uma criança antes da idade de maturação sexual, ou seja, antes dos 8 anos no sexo feminino e 9 anos no sexo masculino ${ }^{(1)}$. Quando dependente de gonadotrofina, é chamada de puberdade precoce verdadeira ou central, com o desenvolvimento dos caracteres sexuais secundários decorrendo da ativação prematura do eixo hipotálamo hipófise gonadal. Já os casos independentes de gonadotrofina são denominados de pseudopuberdade precoce, sendo resultantes da produção autônoma dos esteroides sexuais ${ }^{(2)}$.

Tendo em vista que 95\% das crianças do sexo feminino com puberdade precoce central (PPC) apresentam etiologia idiopática ${ }^{(3)}$, vários fatores têm sido apontados como potencial de risco na evolução para PPC, os quais estão relacionados a interferências ambientais; distúrbios metabólicos, incluindo a obesidade; e condições familiares, no caso da $\operatorname{adoção(4).~}$

Embora haja uma tendência secular para o início da puberdade mais cedo, a obesidade tem um importante significado nesse processo, como mostram estudos recentes acerca do papel da adiposidade no desenvolvimento puberal mais precoce no sexo feminino ${ }^{(5)}$, sem desconsiderar outros fatores, como polimorfismo genético, nutrição, atividade física e interferências ambientais ${ }^{(6)}$.

O estudo Pediatric Research in Office Setting (PROS) mostrou clara associação entre a obesidade infantil, refletida pelo Índice de Massa Corporal (IMC), e o desenvolvimento puberal precoce em crianças do sexo feminino, brancas, de 6 a 9 anos, em razão da média do escore $z$ do IMC ter sido significativamente maior nestas quando comparadas àquelas em pré-puberdade ${ }^{(7)}$.

Em estudos retrospectivos, crianças do sexo feminino adotadas internacionalmente apresentavam elevado risco de desenvolver puberdade precoce. A primeira observação ocorreu na Suécia, em $1991^{(6)}$, e, em seguida, em estudos realizados na Europa e nos Estados Unidos ${ }^{(8-9)}$. O mecanismo para explicar a relação entre PPC e adoção permanece desconhecido na maioria dos casos; especula-se, contudo, se há influência racial, emocional e de fatores ambientais, bem como a evidente relação entre obesidade na infância e início precoce da puberdade ${ }^{(10)}$.

O pronto diagnóstico e tratamento da $\mathrm{PCC}$, tão $\operatorname{logo}$ surjam os primeiros sinais, é de fundamental importância para interromper a progressão das características sexuais secundárias e menstruação, e promover o bem-estar psicossocial da criança ${ }^{(11)}$.

Sem o diagnóstico e tratamento precoces, crianças em PPC estão em grave risco de iniciação sexual mais cedo, abusos sexuais, avanço da idade óssea acelerada e baixa estatura com obesidade na vida adulta ${ }^{(11)}$, o que resulta em elevado risco de desenvolvimento de hiperinsulinemia ${ }^{(12)}$, diabetes mellitus e doenças cardiovasculares ${ }^{(13)}$.

Sendo uma condição com repercussões a curto, médio e longo prazo no estado de saúde da criança, torna-se de fundamental importância o planejamento e a implementação de ações de promoção à saúde voltadas para as crianças com $\mathrm{PPC}^{(14)}$.

Para isso, faz-se necessário conhecer quem são essas crianças, sendo esse conhecimento o alvo da presente inquietação, que objetiva analisar as características das crianças com PPC, identificando-se tanto suas características clínico-epidemiológicas como os fatores determinantes para o recebimento de uma atenção oportuna à saúde.

\section{MÉTODOS}

Estudo do tipo transversal, retrospectivo, com abordagem analítica, realizado no Hospital Universitário Walter Cantídio (HUWC) da Universidade Federal do Ceará, investigou prontuários de crianças com PPC acompanhadas no ambulatório de endocrinologia do hospital, as quais chegaram a esse serviço através de encaminhamentos realizados por médicos pediatras, procedentes tanto de serviços públicos como privados, da capital e do interior.

A amostra do estudo compreendeu 342 prontuários de crianças de 1 a 11 anos (correspondendo a todos os casos clínicos e/ou laboratoriais de PPC atendidos no período de 1994 a 2010), as quais tiveram início da puberdade antes dos 8 anos de idade para o sexo feminino, e antes de 9 anos para o sexo masculino. As informações contidas nos prontuários foram transpostas, pelo investigador principal e por residentes sob sua supervisão, para um formulário próprio do estudo (protocolo padrão de atendimento de crianças com PPC), no ano de 2010.

Incluíram-se no estudo crianças do sexo feminino com surgimento de mamas antes dos 8 anos de idade, e do sexo masculino com aumento de volume do testículo ou do pênis 
antes dos 9 anos de idade, tendo apresentado diagnóstico laboratorial e de imagem para PPC. Excluíram-se crianças que iniciaram o surgimento do quadro puberal depois dos 8 anos no sexo feminino, e depois dos 9 anos no sexo masculino, bem como aquelas cujo diagnóstico não foi confirmado.

Os dados analisados procederam do protocolo padrão de atendimento de crianças com PPC do Serviço de Endocrinologia do HUWC, no qual foram registrados os dados clínicos/laboratoriais e antropométricos, além das informações básicas sobre a criança e seus familiares. $\mathrm{O}$ preenchimento do protocolo ocorreu de forma manual, no princípio e no final do tratamento, pelo investigador principal e pelos médicos residentes do referido serviço, sendo posteriormente digitalizado.

As crianças atendidas pelo staff do Serviço de Endocrinologia do HUWC, que inclui o investigador principal e os médicos residentes, inicialmente foram submetidas a exame físico na presença dos pais ou responsáveis, sendo, em seguida, submetidas aos exames laboratoriais e de imagem apropriados. O diagnóstico clínico é baseado no surgimento dos caracteres sexuais secundários, nas idades anteriormente descritas.

O diagnóstico laboratorial baseia-se nas dosagens de gonadotrofinas, ou seja, LH (Hormônio Luteinizante) basal > 0,6 UI/L, e após estímulo com o GnRH (Hormônio Liberador de Gonadotrofina), >6,9 UI/L para o sexo feminino e 9,6 UI/L para o sexo masculino ${ }^{(15)}$. Alternativamente, uma dosagem de LH após 1 a 2 horas da primeira aplicação do análogo do $\mathrm{GnRH}$, de ação prolongada acima de 10 $\mathrm{U} / 1$ (método imunofluorométrico), indica $\mathrm{PPC}^{(16)}$. As radiografias de mão e punho não dominantes permitem a avaliação da idade óssea ${ }^{(17)}$. A idade óssea avançada, bem como a imagem ultrassonográfica que revela útero e ovários acima dos valores pré-puberais, complementam o diagnóstico de PPC. Tanto os dados referentes ao diagnóstico clínico como ao laboratorial eram devidamente registrados no prontuário da criança. As medidas antropométricas de peso e estatura das crianças foram aferidas inicialmente por balanças analógicas do tipo Salter ${ }^{\circledR}$, e a partir de 2005, por balanças eletrônicas da marca Toledo ${ }^{\circledR}$.

As principais variáveis do estudo incluíram as características biológicas, socioeconômicas, familiares e nutricionais das crianças. Após a descrição dessas características, identificaram-se, através da aplicação de testes de significância estatística, os fatores associados à adoção, à obesidade e ao tempo de procura por atenção, consideradas neste estudo como as variáveis de maior interesse.

Realizou-se a análise dos dados através do programa SPSS 17.0, sendo as diferenças de características entre as crianças, apresentando ou não cada uma daquelas três condições, avaliadas pelo teste Qui-Quadrado, com valor alfa de $5 \%$.

Esta pesquisa está de acordo com a Resolução 466/12 do Conselho Nacional de Saúde, que trata da ética em pesquisa envolvendo seres humanos, e recebeu aprovação do Comitê de Ética em Pesquisa (COMEPE) do complexo hospitalar da Universidade Federal do Ceará, sob o protocolo $\mathrm{n}^{\circ}$ 027.03.11 O estudo preservou o anonimato de todos os casos estudados e não realizou nenhum tipo de experimento com as crianças atendidas.

\section{RESULTADOS}

Observou-se que $333(97,4 \%)$ crianças com PPC eram do sexo feminino e apenas $9(2,6 \%)$ eram do sexo masculino, apresentando, portanto, uma razão entre os sexos bastante elevada (37:1).

Quanto à cor da pele, encontrou-se que 193 (64,1\%) crianças avaliadas eram pardas e $7(2,3 \%)$ eram negras. Com relação à renda familiar, $145(50,9 \%)$ crianças provinham de famílias com ganho mensal igual ou superior a quatro salários mínimos (Tabela I).

Uma considerável proporção de crianças com PPC era adotada - 25 (7,3\%). Foram observados $14(4,4 \%)$ casos de consanguinidade entre os pais das crianças com PPC. Cerca de $20 \%$ das mães das crianças analisadas, excluindo-se as adotadas, tiveram sua primeira menstruação entre 4 e 11 anos de idade, e um terço dessas crianças possuía familiares com história de puberdade precoce (Tabela I).

$\mathrm{Na}$ avaliação nutricional das crianças, por ocasião da primeira consulta no serviço de endocrinologia, 92 $(27,1 \%)$ apresentavam sobrepeso e $85(25,1 \%)$, obesidade, perfazendo um total de $177(52,2 \%)$ crianças apresentando algum nível de excesso de peso.

A estatura dos pais é um indicador da altura final dos filhos. No presente estudo, $47(78,9 \%)$ pais e $244(75,5 \%)$ mães apresentavam estatura abaixo do parâmetro médio populacional, que é de $173 \mathrm{~cm}$ para os homens e $160 \mathrm{~cm}$ para as mulheres (Tabela I).

Embora $205(60,3 \%)$ crianças estudadas na atual pesquisa tenham manifestado o início da puberdade com 6 anos ou menos de idade, somente 89 (26,4 \%) foram conduzidas pelos pais ao tratamento em serviço especializado ainda dentro dessa faixa de idade. Nesse sentido, o retardo na procura por atenção verificou-se de forma generalizada, com somente 95 (28\%) crianças recebendo atenção especializada na mesma idade do surgimento dos primeiros sinais de puberdade. Noventa crianças $(26,8 \%)$ receberam assistência especializada somente após dois anos da percepção pelos pais dos primeiros sinais de puberdade (Tabela I). 
Tabela I - Características biológicas, familiares, socioeconômicas e de atenção endocrinológica de crianças com puberdade precoce central (PPC) atendidas em hospital universitário de referência. Fortaleza-CE, 1994-2010.

\begin{tabular}{|c|c|c|}
\hline Características & $\mathbf{n}$ & $\%$ \\
\hline \multicolumn{3}{|l|}{ Sexo } \\
\hline Feminino & 333 & 97,4 \\
\hline Masculino & 9 & 2,6 \\
\hline \multicolumn{3}{|l|}{ Cor da pele } \\
\hline Branca & 99 & 32,9 \\
\hline Negra & 7 & 2,3 \\
\hline Parda & 193 & 64,1 \\
\hline Amarela & 2 & 0,7 \\
\hline \multicolumn{3}{|l|}{ Origem da crianca } \\
\hline Biológica & 317 & 92,7 \\
\hline Adotada & 25 & 7,3 \\
\hline \multicolumn{3}{|l|}{ Estado nutricional da criança* } \\
\hline Eutrófico & 162 & 47,8 \\
\hline Sobrepeso & 92 & 27,1 \\
\hline Obesidade & 85 & 25,1 \\
\hline \multicolumn{3}{|l|}{ Renda familiar } \\
\hline Até 1 salário mínimo & 22 & 7,7 \\
\hline 2 salários mínimos & 49 & 17,2 \\
\hline 3 salários mínimos & 69 & 24,2 \\
\hline 4 salários mínimos ou mais & 145 & 50,9 \\
\hline \multicolumn{3}{|l|}{ Consanguinidade dos pais } \\
\hline Sim & 14 & 4,4 \\
\hline Não & 303 & 95,6 \\
\hline \multicolumn{3}{|l|}{ Menarca da mãe } \\
\hline $4-11$ anos & 59 & 19,5 \\
\hline 12 anos & 92 & 30,5 \\
\hline $13 \mathrm{ou}+\operatorname{anos}$ & 151 & 50,0 \\
\hline \multicolumn{3}{|c|}{ História familiar de puberdade precoce } \\
\hline Sim & 92 & 31,0 \\
\hline Não & 205 & 69,0 \\
\hline \multicolumn{3}{|l|}{ Altura do pai } \\
\hline$<1,73$ & 247 & 78,9 \\
\hline$\geq 1,73$ & 66 & 21,1 \\
\hline \multicolumn{3}{|l|}{ Altura da mãe } \\
\hline$<1,60$ & 244 & 75,5 \\
\hline$\geq 1,60$ & 79 & 24,5 \\
\hline \multicolumn{3}{|c|}{ Idade ao início da puberdade** } \\
\hline$\leq 5$ anos & 92 & 27,1 \\
\hline$\overline{6}$ anos & 113 & 33,2 \\
\hline 7 anos & 114 & 33,5 \\
\hline 8 anos & 20 & 5,9 \\
\hline$\geq 9$ anos & 1 & 0,3 \\
\hline \multicolumn{3}{|l|}{ Idade na $1^{\text {a }}$ consulta } \\
\hline$\leq 5$ anos & 30 & 8,8 \\
\hline$\overline{6}$ anos & 60 & 17,6 \\
\hline 7 anos & 105 & 30,8 \\
\hline 8 anos & 117 & 34,3 \\
\hline$\geq 9$ anos & 29 & 8,5 \\
\hline \multicolumn{3}{|l|}{ Años entre $1^{\circ}$ sinal e $1^{a}$ consulta } \\
\hline 0 & 95 & 28,0 \\
\hline 1 ano & 153 & 45,1 \\
\hline 2 anos & 61 & 18,0 \\
\hline 3 anos & 17 & 5,0 \\
\hline 4 anos ou mais & 13 & 3,8 \\
\hline
\end{tabular}

*Baseado no percentil do IMC por ocasião da $1^{\text {a }}$ consulta

** Baseado na época da percepção materna dos sinais característicos 
Na Tabela II, os fatores associados à adoção, especificamente em crianças com puberdade precoce, são identificados. Verificou-se que $18(81,8 \%)$ crianças adotadas procediam de famílias com renda mensal elevada, ou seja, acima de quatro salários mínimos, enquanto no grupo de crianças não adotadas somente 127 (48,3\%) provinham de famílias com poder aquisitivo mais elevado $(\mathrm{p}=0,023)$.

A cor parda da pele apresentou-se mais frequente em crianças adotadas $-15(71,4 \%)$ - do que em filhos biológicos
- $178(63,5 \%)$-, embora essa diferença tenha se situado apenas próxima da significância estatística $(\mathrm{p}=0,083)$.

Casos de PPC se manifestando nos cinco primeiros anos de vida ocorreram com bastante frequência em filhos biológicos - $91(28,9 \%)$-, enquanto em filhos adotivos ocorreu em apenas um caso (4\%).

Não se observou associação entre adoção e sobrepeso/ obesidade nas crianças com PPC. Entre as crianças adotadas,

Tabela II - Associação entre adoção e fatores biológicos, familiares, socioeconômicos e de atenção endocrinológica em crianças com puberdade precoce central (PPC) atendidas em hospital universitário de referência. Fortaleza-CE, 1994-2010.

\begin{tabular}{|c|c|c|c|}
\hline \multirow[b]{2}{*}{ Características } & \multicolumn{2}{|c|}{ Adoção } & \multirow[b]{2}{*}{$p$} \\
\hline & $\begin{array}{c}\text { Adotivos } \\
\text { n }(\%)\end{array}$ & $\begin{array}{c}\text { Biológicos } \\
\text { n }(\%)\end{array}$ & \\
\hline \multicolumn{4}{|l|}{ Sexo } \\
\hline Masculino & $1(4,0)$ & $8(2,5)$ & \multirow[t]{2}{*}{0,499} \\
\hline Feminino & $24(96,0)$ & $309(97,5)$ & \\
\hline \multicolumn{4}{|l|}{ Cor da pele } \\
\hline Branca & $4(19,0)$ & $95(33,9)$ & \multirow{4}{*}{0,083} \\
\hline Parda & $15(71,4)$ & $178(63,5)$ & \\
\hline Negra & $2(9,5)$ & $5(1,8)$ & \\
\hline Amarela & $0(0,0)$ & $2(0,7)$ & \\
\hline \multicolumn{4}{|l|}{ Percentil IMC } \\
\hline Eutrófico & $10(40,0)$ & $152(48,4)$ & \multirow[t]{3}{*}{0,715} \\
\hline Sobrepeso & $8(32,0)$ & $84(26,8)$ & \\
\hline Obeso & $7(28,0)$ & $78(24,8)$ & \\
\hline \multicolumn{4}{|l|}{ Idade no início da puberdade } \\
\hline$\leq 5$ & $1(4,0)$ & $91(28,9)$ & \multirow[t]{5}{*}{0,086} \\
\hline 6 & $11(44,0)$ & $102(32,4)$ & \\
\hline 7 & $12(48,0)$ & $102(32,4)$ & \\
\hline 8 & $1(4,0)$ & $19(6,0)$ & \\
\hline$\geq 9$ & $0(0,0)$ & $1(0,3)$ & \\
\hline \multicolumn{4}{|l|}{ Idade na $1^{a}$ consulta } \\
\hline$\leq 5$ & $0(0,0)$ & $30(9,5)$ & \multirow[t]{5}{*}{0,548} \\
\hline$\overline{6}$ & $4(16,0)$ & $56(17,7)$ & \\
\hline 7 & $9(36,0)$ & $96(30,4)$ & \\
\hline 8 & $9(36,0)$ & $108(34,2)$ & \\
\hline$\geq 9$ & $3(12,0)$ & $26(8,2)$ & \\
\hline \multicolumn{4}{|l|}{ Tempo para diagnóstico } \\
\hline 0 & $9(36,0)$ & $86(27,4)$ & \multirow{5}{*}{0,673} \\
\hline 1 & $10(40,0)$ & $143(45,5)$ & \\
\hline 2 & $4(16,0)$ & $57(18,2)$ & \\
\hline 3 & $2(8,0)$ & $15(4,8)$ & \\
\hline $4 \mathrm{ou}+$ & $0(0,0)$ & $13(4,1)$ & \\
\hline \multicolumn{4}{|l|}{ Renda familiar mensal } \\
\hline Até 1 salário mínimo & $0(0,0)$ & $22(8,4)$ & \multirow{4}{*}{0,023} \\
\hline 2 salários mínimos & $2(9,1)$ & $47(17,9)$ & \\
\hline 3 salários mínims & $2(9,1)$ & $67(25,5)$ & \\
\hline 4 salários mínimos ou mais & $18(81,8)$ & $127(48,3)$ & \\
\hline
\end{tabular}


$15(60 \%)$ apresentavam obesidade/sobrepeso, contra 162 $(51,6 \%)$ entre as crianças não adotivas $(\mathrm{p}=0,715)$.

Com relação aos fatores associados ao estado nutricional de crianças com PPC, observou-se a menarca precoce materna fortemente correlacionada ao nível de sobrepeso/obesidade das crianças $(p=0,011)$. Enquanto 39 $(25 \%)$ crianças com excesso de peso eram filhas de mães que menstruaram com menos de 12 anos de idade, entre as crianças eutróficas, somente 19 (13,3\%) tinham mães relatando tal condição.

A renda familiar, por sua vez, não se mostrou como um fator associado ao sobrepeso/obesidade em crianças com PPC ( $p=0,291)$ (Tabela III).

Ao analisar o tempo entre os primeiros sinais de puberdade e a assistência recebida (Tabela IV), verificouse que, entre as crianças com atraso de mais de um ano para receberem atendimento especializado, mais da metade - $47(51,7 \%)$ - iniciou suas manifestações puberais antes dos 5 anos de idade. Já entre as mais prontamente atendidas, ou seja, as que levaram menos de um ano para o atendimento, somente $45(18,1 \%)$ apresentaram tal padrão acentuadamente precoce da puberdade, com início antes dos 5 anos $(\mathrm{p}<0,001)$.

Entre as crianças atendidas somente após dois anos da percepção dos primeiros sinais puberais por parte dos pais, $13(17,8 \%)$ eram provenientes de famílias com renda mensal de até 1 salário mínimo; já entre aquelas atendidas dentro do primeiro ano após o aparecimento dos sintomas, o percentual foi de somente 4,3\% (9) $(\mathrm{p}=0,002)$.

Ainda sobre o retardo no atendimento, enquanto entre as crianças atendidas dentro do primeiro ano mais de um terço apresentava um quadro puberal menos avançado, com somente um sinal sendo detectado, entre aquelas atendidas com dois anos ou mais, somente $14(16,1 \%)$ apresentavam quadro inicial com um único sinal de puberdade $(\mathrm{p}=0,048)$.

Tabela III - Associação entre estado nutricional, fatores biológicos, familiares e socioeconômicos em crianças com puberdade precoce central (PPC) atendidas em hospital universitário de referência. Fortaleza-CE, 1994-2010.

\begin{tabular}{|c|c|c|c|}
\hline \multirow[b]{2}{*}{ Características } & \multicolumn{2}{|c|}{ Estado nutricional } & \multirow[b]{2}{*}{$p$} \\
\hline & $\begin{array}{l}\text { Sobrepeso/Obeso } \\
\text { n (\%) }\end{array}$ & $\begin{array}{c}\text { Eutrófico } \\
\text { n (\%) }\end{array}$ & \\
\hline \multicolumn{4}{|l|}{ Sexo } \\
\hline Masculino & $6(3,4)$ & $3(1,9)$ & \multirow[t]{2}{*}{0,506} \\
\hline Feminino & $171(96,6)$ & $159(98,1)$ & \\
\hline \multicolumn{4}{|l|}{ Amamentação } \\
\hline Foi amamentado & $122(76,7)$ & $109(74,1)$ & \multirow[t]{2}{*}{0,600} \\
\hline Não foi amamentado & $37(23,3)$ & $38(25,9)$ & \\
\hline \multicolumn{4}{|l|}{ Renda familiar mensal } \\
\hline Até 1 salário mínimo & $11(7,5)$ & $11(8,1)$ & \multirow{4}{*}{0,291} \\
\hline 2 salários mínimos & $25(17,0)$ & $24(17,8)$ & \\
\hline 3 salários mínimos & $37(25,2)$ & $31(23,0)$ & \\
\hline 4 salários mínimos ou mais & $74(50,3)$ & $69(51,1)$ & \\
\hline \multicolumn{4}{|l|}{ Origem da criança } \\
\hline Biológica & $162(91,5)$ & $152(93,8)$ & \multirow{2}{*}{0,418} \\
\hline Adotada & $15(8,5)$ & $10(6,2)$ & \\
\hline \multicolumn{4}{|l|}{ Menarca da mãe } \\
\hline 4-11 anos & $39(25,0)$ & $19(13,3)$ & \multirow{3}{*}{0,011} \\
\hline 12 anos & $50(32,1)$ & $41(28,7)$ & \\
\hline $13 \mathrm{ou}+\operatorname{anos}$ & $67(42,9)$ & $83(58,0)$ & \\
\hline
\end{tabular}


Tabela IV - Associação entre o tempo até o diagnóstico e fatores biológicos, socioeconômicos e achados clínicos em crianças com puberdade precoce central (PPC) atendidas em hospital universitário de referência. Fortaleza-CE, 1994-2010.

\begin{tabular}{|c|c|c|c|}
\hline \multirow[b]{2}{*}{ Características } & \multicolumn{2}{|c|}{ Tempo decorrido até o diagnóstico } & \multirow[b]{2}{*}{$p$} \\
\hline & $\begin{array}{c}\text { Maior que } 1 \text { ano } \\
\text { n (\%) }\end{array}$ & $\begin{array}{c}\text { Até } 1 \text { ano } \\
\text { n (\%) }\end{array}$ & \\
\hline \multicolumn{4}{|l|}{$\overline{\text { Cor da pele }}$} \\
\hline Branca & $33(40,2)$ & $65(30,1)$ & \multirow{4}{*}{0,20} \\
\hline Parda & $46(56,1)$ & $145(67,1)$ & \\
\hline Negra & $3(3,7)$ & $4(1,9)$ & \\
\hline Amarela & $0(0,0)$ & $2(0,9)$ & \\
\hline \multicolumn{4}{|l|}{ Idade no início da puberdade } \\
\hline$\leq 5$ & $47(51,7)$ & $45(18,1)$ & \multirow{5}{*}{0,00} \\
\hline$\overline{6}$ & $32(35,2)$ & $81(32,7)$ & \\
\hline 7 & $11(12,1)$ & $102(41,1)$ & \\
\hline 8 & $0(0,0)$ & $20(8,1)$ & \\
\hline$>9$ & $1 .(1,1)$ & $0(0,0)$ & \\
\hline \multicolumn{4}{|l|}{ Estado nutricional } \\
\hline Eutrófico & $42(46,7)$ & $120(48,4)$ & \multirow{3}{*}{0,533} \\
\hline Sobrepeso & $28(31,1)$ & $63(25,4)$ & \\
\hline Obeso & $20(22,2)$ & $65(26,2)$ & \\
\hline \multicolumn{4}{|l|}{ Origem da crianca } \\
\hline Biológica & $85(93,4)$ & $229(92,3)$ & \multirow[b]{2}{*}{0,739} \\
\hline Adotada & $6(6,6)$ & $19(7,7)$ & \\
\hline \multicolumn{4}{|l|}{ Renda familiar mensal } \\
\hline Até 1 salário mínimo & $13(17,8)$ & $9(4,3)$ & \multirow{3}{*}{0,002} \\
\hline 2 a 3 salários mínimos & $28(38,3)$ & $89(42,4)$ & \\
\hline 4 ou mais salários mínimos & $32(43,7)$ & $112(53,5)$ & \\
\hline \multicolumn{4}{|l|}{ Evolução dos sinais } \\
\hline Alterações só das mamas & $12(13,8)$ & $72(30,8)$ & \multirow{7}{*}{0,048} \\
\hline Alterações só dos pelos & $2(2,3)$ & $6(2,6)$ & \\
\hline Alterações só dos testículos & $0(0,0)$ & $1(0,4)$ & \\
\hline Evolução das mamas para pelos & $42(48,3)$ & $99(42,3)$ & \\
\hline Evolução dos pelos para mamas & $21(24,1)$ & $31(13,2)$ & \\
\hline Evolução dos testículos para pelos & $5(5,7)$ & $14(6,0)$ & \\
\hline Evolução dos pelos para testículos & $5(5,7)$ & $11(4,7)$ & \\
\hline
\end{tabular}

\section{DISCUSSÃO}

Embora a ocorrência de PPC em crianças do sexo masculino seja reconhecidamente baixa, observou-se na presente pesquisa uma razão excessivamente elevada entre o sexo feminino e o masculino, de 37:1, bastante acima da observada em revisão da literatura internacional, que encontrou razões situadas entre 3:1 e 23:1(18). Essa observação sugere um possível desconhecimento por parte dos pais das crianças quanto ao momento inicial do ingresso da puberdade para as crianças do sexo masculino, o que poderia implicar em uma menor procura por atendimento especializado para crianças do sexo masculino com puberdade precoce.

Identificou-se no presente estudo $7,3 \%$ de crianças adotadas com PPC, uma proporção cinco vezes maior do que a observada no estado do Ceará, onde levantamentos populacionais têm mostrado adoção em somente $1,5 \%$ das crianças ${ }^{(19)}$. Estudos mostram que crianças do sexo feminino adotadas internacionalmente têm riscos mais elevados de desenvolver a puberdade mais cedo, quando comparadas com aquelas não adotadas. Um grande estudo belga levantou a hipótese sobre a possível influência de pesticidas ambientais no desenvolvimento da puberdade entre os imigrantes que haviam saído de países em desenvolvimento ${ }^{(20)}$. No entanto, dois outros estudos demonstraram não haver maior risco de desenvolvimento puberal na população infantil que havia imigrado com seus pais para Dinamarca e Espanha ${ }^{(4,21)}$. Os achados do presente estudo, por sua vez, sugerem ser a adoção um elemento causal do desenvolvimento da puberdade mais cedo, independentemente de ela acontecer em âmbito local ou internacional. Desse modo, a PPC das crianças adotadas poderia estar vinculada às questões traumáticas ou emocionais decorrentes do processo de adoção em si, e não relacionada à questão da imigração ou fatores ambientais dos países de origem. 
Comparando filhos adotados e biológicos com PPC, não se observou diferença significativa quanto à presença de obesidade/sobrepeso na presente pesquisa. Esses dados sugerem, com relação à gênese da PPC nas crianças adotadas, não haver influência da obesidade $(\mathrm{p}=0,715)$, que, portanto, estaria eventualmente atuando como um fator de confusão. Esse achado reforça a hipótese de que a adoção constitui um fator de risco independente para a PPC, corroborando com os achados das observações em estudos internacionais $^{(22)}$.

Existe clara associação entre obesidade infantil, refletida pelo IMC, e desenvolvimento puberal precoce, tendo em vista a ação da aromatase, enzima produzida pelo tecido adiposo capaz de fazer a conversão de andrógenos em estrógenos, expondo as crianças pré-púberes, com sobrepeso e obesidade a uma maior carga de estrógeno ${ }^{(10)}$. $\mathrm{Na}$ atual investigação, mais da metade das crianças estavam acima do peso, com 27,1\% apresentando sobrepeso e $25,1 \%$, obesidade. Essa frequência elevada de sobrepeso e obesidade muito provavelmente está relacionada à introdução de alimentos inadequados nos primeiros meses de vida, bem como ao fácil acesso a produtos hipercalóricos e gordurosos por parte das diversas classes sociais. Isso favorece um rápido ganho de peso na primeira infância, com alterações da homeostase hormonal, potencializando uma ocorrência puberal antecipada ${ }^{(23,24)}$.

No presente estudo, a menarca precoce materna esteve associada de modo significativo $(\mathrm{p}=0,011)$ ao nível de sobrepeso/obesidade nas crianças com PPC, com $25 \%$ das crianças obesas sendo filhas de mães que menstruaram com menos de 12 anos, contra 13,3\% das crianças eutróficas. Isso remete a um estudo realizado na região semiárida do Brasil, que mostrou um risco 59\% maior de obesidade em mulheres que apresentaram a menarca antes dos 12 anos de idade, em comparação àquelas que a apresentaram após essa idade ${ }^{(25)}$.

No que se refere à questão estatural, a altura dos pais tem influência decisiva na expectativa da altura de seus filhos, sendo, portanto, um indicador importante na avaliação estatural das crianças ${ }^{(26)}$. Constatou-se no presente estudo que quase $80 \%$ dos pais e das mães das crianças com PPC apresentavam estatura abaixo do parâmetro médio populacional. Sabendo-se que o risco da criança com PPC evoluir com perda estatural na vida adulta é bastante elevado, se seus pais apresentarem estatura abaixo da média populacional, esse agravante assume uma dimensão ainda maior $^{(27)}$.

Quanto menor é a idade de início puberal, maiores são os riscos de tumores como causa de $\mathrm{PPC}^{(28)}$. O início da puberdade aos 6 anos de idade ou menos ocorreu em $60,3 \%$ das crianças estudadas na atual pesquisa. Tendo em vista o risco acarretado por esse início puberal excessivamente precoce, causa preocupação quando $42,8 \%$ dessas crianças só tiveram acesso ao atendimento para investigação e tratamento quando já tinham 8 anos ou mais. É preocupante também constatar que $45,1 \%$ das crianças já haviam iniciado os primeiros sinais de puberdade há pelo menos um ano no momento do atendimento especializado, e $91(26,8 \%)$ haviam levado de 2 a 4 anos para receber um atendimento efetivo.

Assim, ao confrontar-se o tempo entre os primeiros sinais de puberdade e a assistência recebida, constatouse no estudo em questão que quanto mais precoce o aparecimento dos primeiros sinais de puberdade, maior o retardo em se conseguir atendimento especializado. Enquanto entre as crianças com demora de mais de 2 anos para receber o atendimento especializado mais da metade havia iniciado seus quadros puberais antes dos 5 anos de idade, entre aqueles com demora de menos de um ano para o atendimento, somente um quinto iniciou o quadro de PPC com menos de 5 anos. Como consequência desse retardo, observou-se que, entre aquelas atendidas a partir do segundo ano do aparecimento dos sinais puberais, $83,8 \%$ estavam em níveis significativamente mais avançados da puberdade do que as crianças $(66,2 \%)$ atendidas dentro do primeiro ano do aparecimento dos sinais $(\mathrm{p}=0,048)$. Isso mostra como crianças em PPC, quando não tratadas no tempo adequado, sofrem aceleração do processo, exigindo uma maior rapidez nos cuidados, sob pena de maior exposição a conflitos psicológicos, assédio sexual e pior prognóstico para estatura final ${ }^{(27-29)}$.

Um claro indicador de iniquidade é constatado quando se observa que, entre as crianças atendidas somente no segundo ano após seus pais perceberem os primeiros sinais puberais, $17,8 \%$ eram provenientes de famílias com renda mensal de até um salário mínimo, contra $4,3 \%$ das crianças atendidas dentro do primeiro ano após o aparecimento dos sinais $(\mathrm{p}=0,002)$. O presente achado aponta para uma importante influência do fator econômico no acesso ao serviço público especializado em endocrinologia, fato este corroborado em estudo sobre a inequidade no acesso aos cuidados de saúde no Nordeste do Brasil, mostrando uma significativa associação positiva entre a renda familiar e os níveis hierárquicos de atenção à saúde ${ }^{(30)}$.

As limitações do presente estudo são aquelas típicas de estudos transversais, como o problema da casualidade reversa, quando não é possível determinar o que seria exposição ou efeito. Não foi possível também determinar a prevalência da PPC, uma vez que os casos estudados foram provenientes de um serviço, e não da população. Vale salientar, no entanto, que o referido serviço é, entre os existentes no Estado, aquele de maior representação no atendimento a casos de $\mathrm{PPC}$, proporcionando à amostragem do estudo uma considerável representatividade. 


\section{CONCLUSÃO}

Entre as crianças com puberdade precoce central, o estudo encontrou proporções elevadas de adoção e de sobrepeso/obesidade, dois fatores considerados de alto risco para o desencadeamento do problema. Uma elevada proporção dessas crianças sofreu também um significativo retardo na obtenção da atenção especializada, essencial para a interrupção do processo puberal em curso. Constatou-se que esse retardo ocorre principalmente quando a criança manifesta um aparecimento excessivamente precoce dos sinais de puberdade e quando procede de uma família de muito baixa renda. Desse modo, recomenda-se a capacitação de profissionais formais e não formais de saúde para a detecção de sinais precoces de puberdade, com o pronto encaminhamento dos casos, especialmente em populações socioeconomicamente desfavorecidas.

\section{AGRADECIMENTOS}

Ao Programa de Pós-Graduação em Saúde Pública da Universidade Federal do Ceará.

Declaração: os autores declaram não haver conflitos de interesse científico neste estudo.

\section{REFERÊNCIAS}

1. Garibaldi L, Chemaitilly W. disorders of pubertal development. In: Kliegman RM, Stanton BF, St. Geme J, Behrman RE, editors. Nelson textbook of pediatrics. $19^{\text {th }}$ ed. Philadelphia: Saunders Elsevier; 2011. p. 18867.

2. Styne DM, Grumbach MM. Puberty: ontogeny, neuroendocrinology, physiology, and disorders. In: Melmed S, Polonsky KS, Larsen PR, Kronenberg HM, editors. Williams textbook of endocrinology. $12^{\text {th }}$ ed. Philadelphia: Saunders; 2011. p. 1054-201.

3. Giabicani E, Allali S, Durand A, Sommet J, CoutoSilva A-C, Brauner R. Presentation of 493 consecutive girls with idiopathic central precocious puberty: a single-center study. Plos One. 2013;8(7):e70931.

4. Teilmann G, Pedersen CB, Skakkebaek NE, Jensen TK. Increased risk of precocious puberty in internationally adopted children in Denmark. Pediatrics. 2006;118(2):391-9.

5. Rosenfield RL, Lipton RB, Drum ML. Thelarche, pubarche, and menarche attainment in children with normal and elevated body mass index. Pediatrics. 2009;123(1):84-8.
6. Atay Z, Turan S, Guran T, Furman A, Bereket A. Puberty and influencing factors in schoolgirls living in Istanbul: end of the secular trend? Pediatrics. 2011;128(1):40-5.

7. Kaplowitz PB, Oberfield SE. Reexamination of the age limit for defining when puberty is precocious in girls in the United States: implications for evaluation and treatment. Pediatrics. 1999;104(4):936-41.

8. Proos LA, Hofvander Y, Tuvemo T. Menacheal age and growth pattern of Indian girls adopted in Sweden.I. Menarcheal age. Acta Paediatr Scand. 1991;80(89):852-8.

9. Mason P, Narad C. Growth and pubertal development in internationally adopted children. Curr Opin Endocrinol Diabetes. 2002;9(1):26-31.

10. Ahmed ML, Ong KK, Dunger DB. Childhood obesity and the timing of puberty. Trends Endocrinol Metab. 2009;20(5):237-42.

11. Golub MS, Collman GW, Foster PM, Kimmel CA, Rajpert-De Meyts E, Reiter EO, et al. Public health implications of altered puberty timing. Pediatrics. 2008;121(Suppl 3):S218-30.

12. Frontini MG, Srinivasan SR, Berenson GS. Longitudinal changes in risk variables underlying metabolic syndrome $\mathrm{X}$ from childhood to young adulthood in female subjects with a history of early menarche: the Bogalusa Heart Study. Int J Obes Relat Metab Disord. 2003;27(11):1398-404.

13. Pierce MB, Kuh D, Hardy R. Role of lifetime body mass index in the association between age at puberty and adult lipids: findings from men and women in a British birth cohort. Ann Epidemiol. 2010;20(9):67682.

14. Berberoglu M. Precocious puberty and normal variant puberty: definition, etiology, diagnosis and current management. J Clin Res Pediatr Endocrinol. 2009;1(4):164-74.

15. Brito VN, Batista MC, Borges MF, Latronico AC, Kohek MB, Thirone AC, et al. Diagnostic value of fluorometric assays in the evaluation of precocious puberty. J Clin Endocrinol Metab. 1999;84(10):353944.

16. Brito VN, Latronico AC, Arnhold IJ, Mendonça BB. A single luteinizing hormone determination 2 hours after depot leuprolide is useful for therapy monitoring of gonadotropin-dependent precociou puberty in girls. J Clin Endocrinol Metab. 2004;89(9):4338-42. 
17. Greulich WW, Pyle SI. Radiographic atlas of skeletal development of the hand and wrist. Stanford: Stanford University Press; 1959.

18. Partsch CJ, Heger S, Sippell WG. Management and outcome of central precocious puberty. Clin Endocrinol. 2002;56(2):129-48.

19. Secretaria Estadual de Saúde do Ceará. Relatório da V Pesquisa sobre Saúde Materno-Infantil do Ceará (PESMIC V). Fortaleza: SESA; 2008.

20. Krstevska-Konstantinova M, Charlier C, Craen M, Du Caju M, Heinrichs C, de Beaufort C, et al. Sexual precocity after immigration from developing countries to Belgium: evidence of previous exposure to organochlorine pesticides. Hum Reprod. 2001;16(5):1020-6.

21. Soriano-Guillén L, Corripio R, Labarta JI, Cañete R, Castro-Feijóo L, Espino R, et al. Central precocious puberty in children living in Spain: incidence, prevalence, and influence of adoption and immigration. J Clin Endocrinol Metab. 2010;95(9):4305-13.

22. Hayes P. International adoption, "early" puberty, and underrecorded age. Pediatrics. 2013;131(6):1029-31.

23. Silva IS, De Stavola BL, Mann V, Kuh D, Hardy R, Wadsworth ME. Prenatal factors, childhood growth trajectories and age at menarche. Int $\mathrm{J}$ Epidemiol. 2002;31(2):405-12.

24. Biro FM, Greenspan LC, Galvez MP. Puberty in girls of the 21st century. J Pediatr Adolesc Gynecol. 2012;25(5):289-94.

25. Correia LL, Silveira DMI, Silva AC, Campos JS, Machado MMT, Rocha HAL, et al. Prevalência e determinantes de obesidade e sobrepeso em mulheres em idade reprodutiva residentes na região semiárida do Brasil. Ciênc Saúde Coletiva. 2011;16(1):133-45.

26. Seaver LH, Irons M. ACMG practice guideline: genetic evaluation of short stature. Genet Med. 2009;11(6):46570

27. Wit JM, Balen HV, Kamp GA, Oostdijk W. Benefit of postponing normal puberty for improving final height. Eur J Endocrinol. 2004;151(Suppl 1):S41-5.

28. Choi KH, Chung SJ, Kang MJ, Yoon JY, Lee JE, Lee YA, et al. Boys with precocious or early puberty: incidence of pathological brain magnetic resonance imaging findings and factors related to newly developed brain lesions. Ann Pediatr Endocrinol Metab. 2013;18(4):183-90.

29. Lazar L, Meyerovitch J, de Vries L, Phillip M, Lebenthal Y. Treated and untreated women with idiopathic precocious puberty: long-term follow-up and reproductive outcome between the third and fifth decades. Clin Endocrinol. 2014;80(4):570-6.

30. Garcia-Subirats I, Vargas I, Mogollón-Pérez AS, Paepe $P$, Silva MRF, Unger JP, et al. Inequities in access to health care in different health systems: a study in municipalities of central Colombia and north-eastern Brazil. Int J Equity Health. 2014;13(10):1-15.

\section{Endereço para correspondência:}

Celso Junior Wanderley Cavalcante

Hospital Universitário Walter Cantídio - UFC

Rua Capitão Francisco Pedro, 1290

Bairro: Rodolfo Teófilo

CEP: 60430-370 - Fortaleza - CE - Brasil

E-mail: cjwcavalcante@gmail.com 